\title{
Encrassement et nettoyage d'un échangeur de chaleur à plaques lors du traitement UHT d'un produit lacté chocolaté. Comparaison avec le lait
}

\author{
F. RENE *, J.C. LEULIET **, M. GOLDBERG *, M. LALANDE ** \\ * B.S.N., Centre International de Recherche Daniel Carasso, \\ 92350 Le Plessis Robinson, France \\ * I.N.R.A., Laboratoire de Génie industriel alimentaire, \\ B.P. 39, 59651 Villeneuve d'Ascq Cedex, France
}

\section{Résumé}

L'étude de l'encrassement d'un échangeur de chaleur à plaques (modèle VICARB V7) pour la fabrication de crèmes dessert (parfum chocolat) a été réalisée. Au cours de trois essais, l'évolution de l'encrassement des différentes zones d'échange a été suivie en continu. L'échangeur a été systématiquement démonté et la masse de dépôt frais adhérant aux plaques a été mesurée afin de connaître la répartition interne de ces dépôts le long du chemin thermique. Une plaque sur deux a été conservée et les dépôts récupérés afin d'être analysés. L'échangeur a ensuite été remonté en substituant aux plaques prélevées d'autres propres. Trois séquences de nettoyage ont donc pu être testées ensuite, en modifiant à chaque fois le produit de la phase alcaline.

Les résultats obtenus concernant l'encrassement sont comparés à ceux que l'on obtient lors de la stérilisation UHT du lait. La similitude des compositions et répartitions en masse est présentée et discutée. Pour ce qui concerne le nettoyage, la difficulté d'élimination des dépôts formés par cette préparation chocolatée a été une nouvelle fois mise en évidence. Les améliorations apportées par l'utilisation de produits de nettoyage spécifiques ont été vérifiées après ouverture de l'appareil.

La présence de composés tels que l'amidon, le cacao et le chocolat dans ce type de préparation rend délicate la transposition des modèles d'interprétation du phénomène établis dans le cas du lait.

Mots clés: Encrassement - Nettoyage - Echangeur de chaleur à plaques - Desserts chocolatés.

\section{Summary}

Fouling and cleaning study of a plate heat exchanger used for chocolated desserts processing

The study of heat exchanger surfaces fouled by cream based desserts preparation was carried out on a VICARB V7 type exchanger. Three trials were made. The fouling evolution was measured on line using the method described by LALANDE and CORRIEU 
(1979). The exchanger was opened each time and the wet weight of deposits adhering to the plates was measured in order to establish inside repartition of deposits along the temperature profile. One plate out of two was removed and the deposit was collected and analysed (protein, minerals, fat and carbohydrates content). Then the exchanger was set up with cleaned plates in place of removed ones. Three cleaning tests were made with different alkaline products. Better cleaning, as observed after exchanger opening was obtained when specific cleaning products are used. Two trials were then performed on an industrial exchanger in order to quantify cleaning efficiency of these products.

Results were compared with those obtained during UHT treatment of milk. Similarity in composition of deposits and mass repartition is presented and discussed. The difficulty of removing soils from surfaces with cleaning in place was observed once again.

Compounds like starch, cocoa and chocolate in the mixture certainly interact between them, so interpretative models developed for milk fouling need to be used carefully in this case.

Key words: Fouling - Cleaning - Plate heat exchanger - Chocolated based desserts.

\section{Introduction}

Depuis plusieurs années, les échangeurs de chaleur à plaques sont utilisés dans le secteur agro-alimentaire, et notamment dans le domaine de la fabrication des produits laitiers frais. Les raisons de ce choix sont dues aux nombreux avantages qu'ils présentent par rapport aux échangeurs tubulaires (HARIOT. 1971 ; Cooper, 1974 ; Vinconneau et Crevel, 1976 ; Lalande et al., 1979). Ils conduisent, en effet, à d'excellents rapports surface d'échange/volume qui limitent leur encombrement et simplifient leur intégration aux unités de production. La modification de leur configuration est aisée ce qui permet de les adapter facilement à un programme thermique différent de celui initialement prévu. L'évolution des produits laitiers frais (yaourts, crèmes dessert, laits gélifiés...) sur le marché nécessite de telles modifications afin d'adapter les procédés de fabrication aux qualités demandées pour ces produits. De même, l'augmentation du coût de l'énergie a entraîné celle des quantités de chaleur récupérées.

Trois causes principales, liées à la conception des échangeurs à plaques, expliquent qu'ils fournissent de meilleurs coefficients généraux d'échange que les échangeurs tubulaires et, par conséquent, qu'ils exigent pour un programme thermique donné, des surfaces d'échange moindres (LALANDE et al., 1979). II s'agit :

- des faibles valeurs de diamètre hydraulique nominal qui se situent entre 5 et $10 \mathrm{~mm}$ alors que des tubes de diamètre inférieur à $20 \mathrm{~mm}(3 / 4$ de pouce) ne sont jamais utilisés industriellement ;

- de la faible épaisseur des parois d'échange que les techniques actuelles d'emboutissage permettent d'obtenir. Elles sont comprises entre 0,6 et $0,8 \mathrm{~mm}$ pour les plaques contre 1 à 1,5 pour des tubes ;

- de l'augmentation de turbulence, à débit constant, provoquée par la structure ondulée des plaques qui conduit à des coefficients de films améliorés. 
Toutefois, leur utilisation de plus en plus répandue pour la réalisation des traitements thermiques pose des problèmes techniques difficiles à résoudre notamment dans l'industrie laitière. Les perturbations des régimes thermique et hydrodynamique dues aux propriétés encrassantes du lait (BurTon, 1968 ; LUND et BIXBY, 1975 ; LALANDE et al., 1979) et aux mélanges complexes tels que les préparations pour crèmes dessert (RENE, 1985) réduisent les temps de fonctionnement du matériel entre deux nettoyages et accroissent les coûts d'exploitation. Les opérations de nettoyage sont délicates à mettre au point et souvent coûteuses puisqu'elles conduisent au démontage des installations pour être terminées manuellement. Dans un souci d'amélioration de ces opérations, il s'avère nécessaire d'approfondir nos connaissances des phénomènes liés à l'encrassement des surfaces au contact des produits laitiers en cours de traitement thermique (Lund et Bixby, 1975 ; LALANDE $e t$ al., 1984 ; Lalande $e t$ al., 1985 ; RoIGNANT et al., 1986 ; HidDink et al., 1986) et de déterminer la nature et la composition des dépôts adhérant sur ces parois (LySTER, 1965; Tissier et $a l ., 1984$; MaAs et al., 1985). Une voie d'étude est l'expérimentation dans des conditions réelles à l'échelle semi-industrielle ou pilote, permettant de connaître l'évolution des compositions ainsi que la répartition en masse des dépôts le long du chemin thermique qu'a suivi le produit au cours de son traitement. Ces résultats permettent d'envisager la simulation du phénomène afin d'essayer d'introduire le critère d'encrassement d'une façon plus adéquate dans les calculs lors du dimensionnement du matériel. Cette approche peut permettre également de s'orienter vers l'emploi d'agents de nettoyage performants vis-àvis du type de souillure considéré.

Nous présentons ici la méthodologie et les résultats que nous avons obtenus lors de la fabrication d'une crème dessert chocolatée ayant une forte tendance à encrasser les surfaces d'échange et qui rend le matériel difficilement nettoyable. Ces essais ont été réalisés au Laboratoire de Génie Industriel Alimentaire de l'INRA de Villeneuve d'Ascq.

\section{Matériel et méthodes}

\section{A. Description de la plate-forme expérimentale}

La plate-forme expérimentale est représentée schématiquement figure 1. Elle comporte essentiellement :

- Deux réservoirs d'une capacité de 2600 litres munis d'un système d'agitation et de régulation de température. L'un est utilisé comme réserve d'eau osmosée pour mise en température de l'installation, l'autre contient la préparation pour crème dessert.

- Un bac de lancement, muni de différents contrôles de niveau, alimente une pompe centrifuge assurant la circulation des fluides dans la première partie du circuit,

- Un préchauffeur à plaques VICARB (modèle V7) assure la montée à température de chambrage de la préparation.

- Un homogénéisateur MANTON GAULIN type M3 utilisé pour fixer le débit de circulation du produit dans l'ensemble de l'installation. 


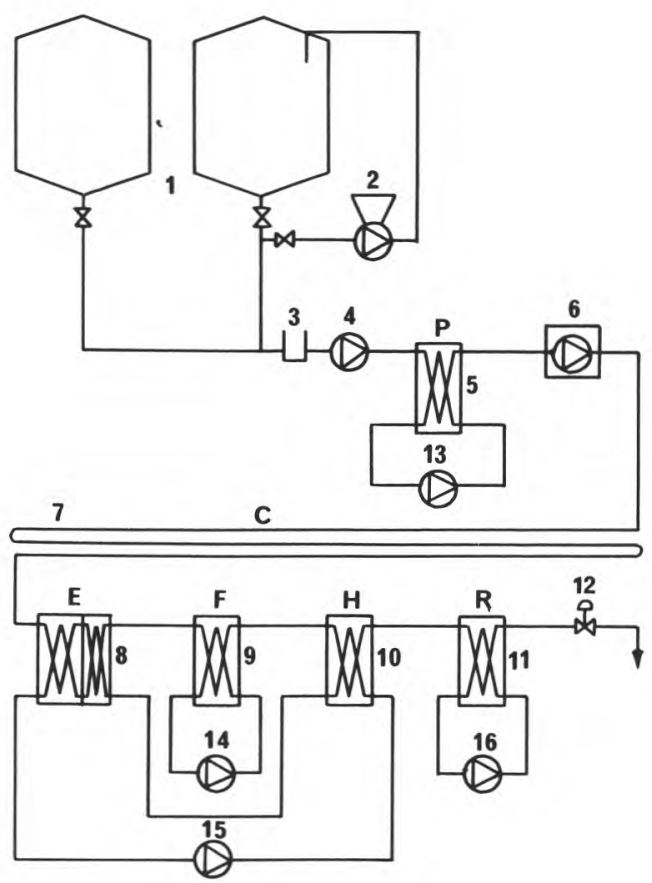

Fig. 1

Schéma de la plate-forme expérimentale

1. Tanks de stockage réfrigérés - 2. Poudreuse - 3. Bac de lancement - 4. Pompe de gavage - 5. Préchauffeur - 6. Homogénéisateur à débit régulé (utilisé sans pression d'homogénéisation) - 7. Chambreur tubulaire - 8. Section de chauffage - 9. Section de stérilisation -10 . Section de récupération -11 . Refroidisseur -12 . Vanne manuelle de contre-pression - 13. Boucle d'eau chaude - 14. Boucle d'eau surchauffée - 15. Boucle d'eau de récupération d'énergie - 16. Boucle d'eau froide

Pilot plant flow diagram

1. Refrigerated tanks of storage - 2. Powders incorporater - 3. Raw tank - 4. Feed pump - 5. Preheater - 6. Homogenizer with regulated flow (used without homogenization pressure) - 7. Holding tube - 8. Heating section - 9. Sterilization section 10. Regeneration section - 11. Cooler - 12. Manuel counter pressure valve - 13. Hot water - 14. Superheated water - 15. Water for heat regeneration -16 . Cold water 
- Un stérilisateur à plaques VICARB, décrit en détail ci-dessous.

- Un refroidisseur à plaques VICARB assure le refroidissement du produit jusqu'à sa température de conditionnement.

- Trois systèmes de production d'eau chaude fonctionnant en circuit fermé et alimentés en calories par injection de vapeur sous contrôle d'un régulateur de température.

- Une réserve d'eau glacée maintenue en froid par un groupe de refroidissement par air.

- Un ensemble de nettoyage en place comprenant trois réservoirs et une pompe de circulation.

- Un système de capteurs de températures, pressions et débits, fournissant pendant l'expérimentation les informations nécessaires, à la surveillance du fonctionnement correct de l'installation et également au suivi en continu du taux de colmatage des différentes sections d'échange par la méthode décrite au paragraphe II.B.

- Une centrale automatique d'acquisition de signaux BURR BROWN MCS 100 A pilotée à distance à l'aide d'un microordinateur BULL Micral 30. Une liaison par boucle de courant permet le dialogue entre la centrale, le microordinateur et un écran clavier AMPEX type 210. Les signaux provenant des capteurs sont transmis par la centrale au microordinateur qui effectue les traitements informatiques nécessaires (conversion en grandeurs physiques, calculs et stockage) et visualise les données sur son écran et sur l'écran clavier placé sur le site.

\section{B. Description du stérilisateur}

L'échangeur utilisé pour la stérilisation est le modèle V7 dont les caractéristiques sont identiques à celles données par LALANDE et al. (1985).

Les zones du stérilisateur où l'encrassement est étudié sont :

- Les sections de récupération de chaleur notées $\mathrm{E}$ et $\mathrm{H}$ dans lesquelles le produit est réchauffé (zones E1 et E2) par une boucle d'eau intermédiaire, qui récupère la chaleur du produit stérilisé en cours de refroidissement (zone $\mathrm{H})$.

- Une section de chauffage notée $\mathrm{F}$ dans laquelle le produit est porté à température de stérilisation par une boucle d'eau surchauffée.

Toutes les sections sont de configuration monocanal (un seul canal par passe) et comportent respectivement :

— zone E1 : 17 passes

- zone E2: 3 passes

- zone $\mathrm{F}: 16$ passes

- zone $\mathrm{H}$ : 10 passes.

\section{Méthodologie expérimentale}

Trois essais ont été réalisés sur l'installation décrite précédemment selon la procédure citée par LALANDE et al. (1984). Ils complètent une série d'essais 
plus importante qui ont été menés sur un appareil industriel au Centre International de Recherches Daniel Carasso (RENE, 1985). La procédure d'essai consiste en plusieurs étapes.

\section{Préparation du mélange en cuve}

Le lait contenu dans la cuve est mis en circulation au moyen d'une pompe centrifuge. Une poudreuse reliée à la tuyauterie permet à l'aide d'une vanne d'y incorporer par aspiration les ingrédients préalablements pesés avec soin (« poudrage »). Il s'agit de poudre de lait, sucre, amidon, cacao, crème et chocolat. Les proportions respectives de ces constituants sont la propriété industrielle du fabricant et ne peuvent pas être révélées. Le mélange contient $73 \%$ de lait et son extrait sec total est de $30 \%$. Le mélange est ensuite laissé sous agitation pendant le temps nécessaire à la bonne incorporation de tous les ingrédients dans le mélange. Les produits préparés pour chaque essai étaient obtenus par incorporation des poudres comme décrit au paragraphe I.A.1. à une quantité de 1000 litres de lait. Ceci nous permettait d'obtenir une durée de fonctionnement d'environ $2,5 \mathrm{~h}$.

\section{Traitement thermique}

Pendant la durée de «poudrage» du lait en cuve, l'installation est stérilisée puis stabilisée à la température de fabrication par passage d'eau osmosée dans le circuit. En basculant les vannes de fond de cuve, on remplace ensuite l'eau osmosée par la préparation. Le produit sortant est récupéré dans des cuves mobiles et une partie est conditionnée manuellement. Une régulation performante, soigneusement mise en ceuvre, permet de maintenir constantes les températures du programme thermique par action sur les températures des boucles d'eau chaude. Pendant toute la durée de fonctionnement, le microordinateur décentralisé effectue à intervalles réguliers le dialogue avec la centrale d'acquisition et l'écran clavier afin de traiter les mesures obtenues par les capteurs. Ces mesures sont sauvegardées sur disque magnétique pour permettre d'effectuer ultérieurement les calculs nécessaires.

Les capteurs de pression implantés aux bornes des sections $\mathrm{E}, \mathrm{F}$ et $\mathrm{H}$ ont permis de suivre en continu l'encrassement des différentes zones au cours des essais. La méthode de suivi (Corriey et al., 1985) a fait l'objet d'un brevet d'invention (LALANDE et CORRIEU, 1979) et est actuellement commercialisée sous forme d'un capteur industriel. La méthode, basée sur la mesure des pertes de charge des sections d'écoulement du produit, a été mise en cuvre dans ce cas à l'aide du microordinateur pilotant à distance la centrale d'acquisition du site. La référence prise dans les calculs est l'écoulement d'eau à travers l'appareil propre dans les conditions de température de l'essai. On suit donc en fonction du temps, l'évolution des différentes pertes de charge de l'échangeur et l'on en déduit un taux de colmatage alpha comme suit :

$$
\alpha=1-\left(\frac{\Delta P_{0}}{\Delta P}\right)^{1 / 3}
$$

où $\Delta \mathrm{P}_{0}$ est la perte de charge de la section propre et $\Delta \mathrm{P}$ la perte de charge de la même section à un instant donné. Les constantes hydrodynamiques du circuit (diamètre hydraulique, loi de frottement) sont d'abord identi- 
fiées sur eau, puis le modèle corrige la valeur de référence $\Delta$ Po en fonction des variations de débit et température. Dès que la cuve de produit est vide, on procède automatiquement au rinçage complet des installations.

Pour chaque essai, le même profil thermique a été respecté dans l'installation (fig. 2). Le débit de fabrication a été fixé à $4001 . \mathrm{h}^{-1}$ et maintenu constant tout au long des essais d'encrassement. Afin d'éviter toute ébullition du produit dans la zone de stérilisation, la pression relative en sortie de zone $\mathrm{H}$ était maintenue à $250 \mathrm{kPa}$ par une vanne de contre-pression manuelle montée sur la tuyauterie en sortie du refroidisseur (fig. 1). Dans ces conditions, le temps de séjour du produit dans le chambreur tubulaire était de $5 \mathrm{mn}$. Les débits d'eau de chauffage étaient de $8101 . \mathrm{h}^{-1}$ pour le préchauffeur, $4001 . \mathrm{h}^{-1}$ pour la section de stérilisation (zone F) et de $7801 . \mathrm{h}^{-1}$ pour la boucle de récupération (zones $\mathrm{E}$ et $\mathrm{H}$ ).

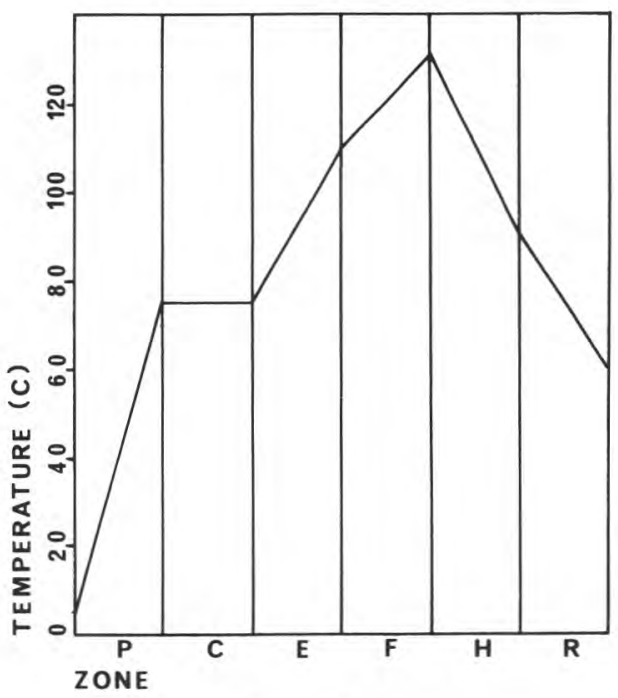

Fig. 2

Profils thermiques suivis par le produit lors des essais

P. Préchauffeur

C. Chambreur

E. Zone de chauffage $E$

$F$. Zone de stérilisation $F$

$H$. Zone de récupération d'énergie $H$

$R$. Refroidisseur

Temperature gradients used during trials

P. Preheater

C. Holding tube

E. Heating section $E$

F. Sterilization section $F$

$H$. Heat regeneration section $H$

R. Cooler 


\section{Démontage du stérilisateur}

Une fois le stérilisateur rincé, ce dernier est démonté. Toutes les plaques sont égouttées et pesées dans des conditions standardisées afin de déterminer le poids de dépôt frais y adhérant. Les plaques numérotées ont fait l'objet de pesées systématiques afin de connaître avec certitude leur masse propre et sèche. Ensuite, une plaque encrassée sur deux est séchée à l'étuve $20 \mathrm{mn}$ à $105^{\circ} \mathrm{C}$ afin d'estimer le poids de dépôt sec correspondant. La totalité de la souillure est alors récupérée et analysée selon ses composants principaux : matières minérales, grasses, protéiques et carbohydratées (voir paragraphe C.4.). Il est possible pour chaque stérilisation de déterminer le profil de répartition de l'encrassement exprimé en poids de produit frais, en poids de produit sec total et en poids de chacun des composants de la souillure. La détermination des poids de dépôts frais ainsi que ceux des dépôts secs se fait par pesée à la balance électronique (METTLER PL 3000) d'une précision de $0,1 \mathrm{~g}$. Raisonnablement, nous pouvons accorder aux mesures une précision de $\pm 0,5 \mathrm{~g}$. Pour les fractions en constituants, la précision est de $1 \%$ sur les fractions protéiques, grasses et carbohydrates et de $0,1 \%$ sur la fraction minérale. Les plaques conservées pour analyse des dépôts sont remplacées par d'autres plaques propres et le stérilisateur est ensuite remonté. On procède alors aux phases de nettoyage puis on démonte l'installation pour estimer l'efficacité des séquences et produits de nettoyage testés.

\section{Analyse des dépôts}

La quantité de protéines totales contenue dans les dépôts a été déterminée d'après la quantité d'azote total $(\times 6,38)$ mesurée par la méthode de Kjeldhal. Après minéralisation en présence d'acide sulfurique, la quantité d'ammoniac dissous est mesurée soit par dosage en retour par une solution titrée de soude soit par électrode spécifique (Helaine, 1977), la quantité d'azote totale est déduite de cette mesure et multipliée par 6,38 pour déterminer la quantité de protéines totales contenue dans les dépôts. Les résultats obtenus sont comparables et de précision équivalente (PAILlER, 1982). La quantité de matière grasse est déterminée selon la méthode de Delsal (1944). La fraction minérale est déterminée par calcination à $550^{\circ} \mathrm{C}$ pendant 16 heures. La fraction carbohydrate a été déterminée par la méthode du réactif phénol-acide sulfurique (Dubors et al., 1956).

\section{Nettoyage des dépôts}

Les problèmes de nettoyage du matériel sont directement liés à la structure des dépôts formés et au mécanisme de croissance de celui-ci (fixation sur une surface puis développement). L'analyse chimique des dépôts peut donner certains renseignements concernant le type de produit de nettoyage à utiliser mais ne représente qu'une partie des éléments permettant d'effectuer un choix rationnel. Actuellement, il est clair que les séquences et produits classiques (acide, soude) sont insuffisants pour éliminer complètement les dépôts présents dans l'appareil. La zone posant le plus de problème est celle de stérilisation dans la gamme de température $110-130{ }^{\circ} \mathrm{C}$. Il semble que la présence de composés tels que l'amidon, les sucres et le chocolat donne au dépôt une structure plus complexe que dans le cas de la stérilisation UHT de lait, ce qui 
le rend difficilement soluble dans des solutions acides ou alcalines. L'échangeur ayant été remonté avec la même configuration que celle utilisée durant le traitement thermique, on procède ensuite aux essais de nettoyage. La moitié des plaques souillées par le produit traité ayant été conservée pour analyser des dépôts, le circuit hydraulique est donc constitué dans chaque canal d'une face propre et d'une face comportant la souillure à éliminer. L'appareil ayant été démonté et la moitié des plaques substituée, l'étalonnage réalisé initialement sur l'appareil propre ne correspond plus aux nouvelles conditions d'écoulement. En effet, la méthode de suivi du taux de colmatage nécessite en premier lieu une identification sur eau de l'échangeur propre qui rend compte de sa géométrie, du type de plaques utilisées (matériaux, ondulations) et du serrage de l'appareil (diamètre hydraulique équivalent). Par conséquent, la méthode de suivi du taux de colmatage décrite au paragraphe C.2. n'a pu être utilisée. La qualité du nettoyage était appréciée visuellement au démontage de l'appareil après passage des séquences de produits acides et alcalins. Le but de ces essais était de comparer l'efficacité des nettoyages. Des recherches précédentes (RENE, 1985) avaient montré la nécessité de tester un produit plus complet que la soude pure. Nous avons donc réalisé pour ces trois essais des procédures de nettoyage identiques en utilisant pour la phase alcaline, différents agents de nettoyage. La procédure était la suivante :

- Rinçage à l'eau osmosée $10 \mathrm{mn}$ et mise en température de l'appareil à $85^{\circ} \mathrm{C}$. Compte tenu des zones de récupération, les températures étaient $75^{\circ} \mathrm{C}$ en entrée de zone $\mathrm{E}, 85^{\circ} \mathrm{C}$ en entrée de zone $\mathrm{F}, 90^{\circ} \mathrm{C}$ en entrée de zone $\mathrm{H}$ et $75^{\circ} \mathrm{C}$ en sortie de zone $\mathrm{H}$.

- Nettoyage acide avec une solution d'acide nitrique à $2 \%(\mathrm{~kg} / \mathrm{kg})$ pendant $20 \mathrm{mn}$ à $85^{\circ} \mathrm{C}(\mathrm{pH}=1)$.

- Rinçage à l'eau osmosée $10 \mathrm{mn}$.

- Nettoyage alcalin pendant $30 \mathrm{mn}$ à $85^{\circ} \mathrm{C}$ avec les produits suivants :

- solution à $2 \%(\mathrm{~kg} / \mathrm{kg})$ d'une poudre alcaline chlorée $(\mathrm{pH}=12,7)$ contenant un complexant du calcium (tripolyphosphates) (Fournisseur HENKEL-FRANCE) ;

- solution à $2 \%(\mathrm{~kg} / \mathrm{kg})$ du même agent alcalin chloré $(\mathrm{pH}=12,7)$ additionnée à $0,5 \%(\mathrm{~kg} / \mathrm{kg})$ d'une poudre libérant en solution de l'oxygène actif (Fournisseur HENKEL-FRANCE).

- Rinçage final à l'eau osmosée et descente en température.

On cherche à déterminer si l'utilisation de produits élaborés se justifie visà-vis des lessives de soude classiques d'emploi très courant.

Chaque phase de nettoyage était effectuée à un débit de $4001 . \mathrm{h}^{-1}$ en boucle ouverte (pas de recyclage sur les bacs de nettoyage), ce qui correspond aux conditions d'étude dans lesquelles ont été effectuées les études du nettoyage des stérilisateurs de lait (PERLAT et al., 1986). L'objectif des essais de nettoyage n'étant pas dans notre cas l'étude cinétique du nettoyage mais un essai d'utilisation. 


\section{Résultats}

\section{A. Analyse des dépôts}

Il apparaît immédiatement que l'encrassement n'est pas réparti de façon homogène dans le sens de la longueur d'échange et qu'il dépend fortement de la zone du stérilisateur considéré. Cet aspect se traduit bien par la figure 3 . On note que l'encrassement est important compte tenu du fait que les volumes de produit à traiter n'excédaient pas $1200 \mathrm{l}$ par essai. Comme dans le cas de la stérilisation du lait (LALANDE et al., 1984), on constate qu'aucune des sections où la température de traitement reste inférieure à $70{ }^{\circ} \mathrm{C}$ ne présente de dépôts solides adhérant aux parois après rinçage. Entre 70 et $110^{\circ} \mathrm{C}$ (sections E1 et E2), la quantité de dépôt formé décroît plus ou moins régulièrement, la répartition étant globalement assez uniforme. Le dépôt, de couleur marron clair, due à la présence du chocolat, a sensiblement le même aspect que celui formé dans cette zone lors du traitement thermique du lait. II est souple, volumineux et spongieux. Sa composition en fraction protéique et minérale est d'ailleurs assez similaire à celle des dépôts du lait dans cette zone de chauffage. On peut considérer que ces fractions sont en moyenne de $55 \%$ de matière protéique, $10 \%$ de matière grasse, $20 \%$ de matière minérale et $15 \%$ de carbohydrates.

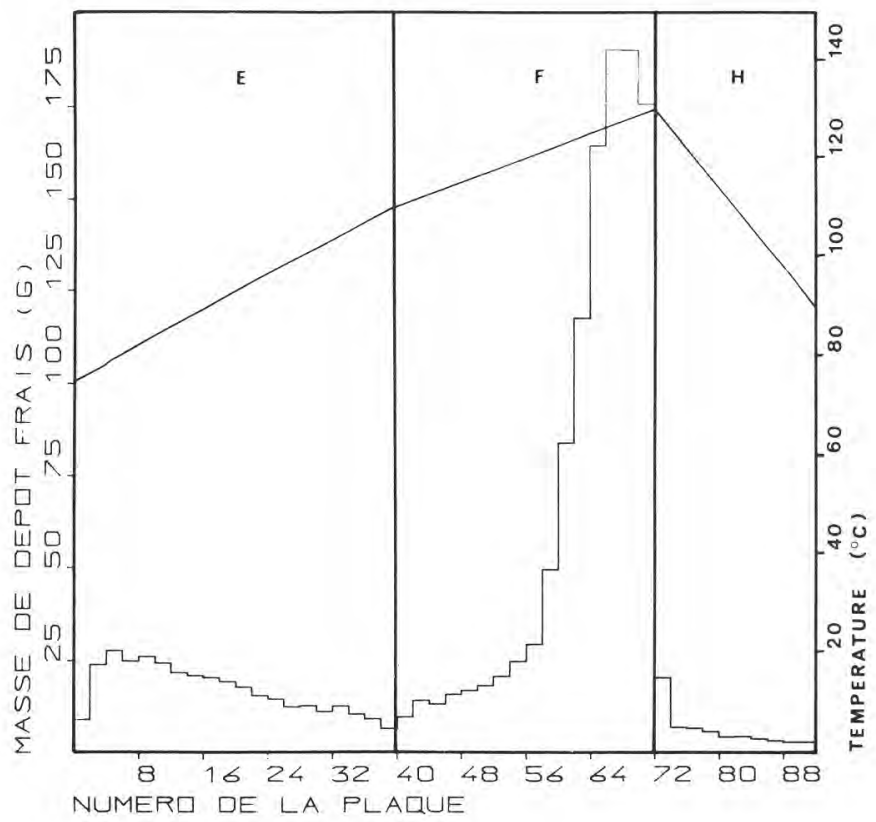

Fig. 3

Masse de dépôts frais par passe obtenus à la fin de l'essai d'encrassement $n^{\circ} 1$.

Wet mass of deposits per pass obtained at the end of the fouling experiment $n^{\circ} 1$. 
Dans la plage de température $110-130^{\circ} \mathrm{C}$, on observe une brusque augmentation de la quantité de dépôt présent sur les plaques. Celle-ci croît rapidement pour passer par un maximum dans la zone $125-130{ }^{\circ} \mathrm{C}$. Le dépôt frais recueilli a un aspect tout à fait particulier ne ressemblant à aucun type de dépôt observé dans le cas du lait (BurTon, 1968 ; Tissier et al., 1984) ou de la crème (Hiddink et al., 1986). De couleur marron foncé, il paraît caoutchouteux et sa structure est élastique. Sa tenue mécanique permet de récupérer des morceaux pouvant atteindre plusieurs dizaines de centimètres carrés. On note par endroits des zones plus foncées ayant un aspect de brûlé au contact de la paroi. Ce dépôt volumineux peut obstruer complètement par endroit l'espace entre plaques. La composition varie de façon sensible avec l'élévation de température (tabl. 1). La fraction protéique du dépôt décroît de $43 \%$ pour les premières plaques de zone $\mathrm{F}$ à $20 \%$ pour les dernières plaques. La fraction minérale est beaucoup plus importante que dans le dépôt recueilli en zone $\mathrm{E}$ : de $45 \%$ en entrée elle passe par un minimum de $21 \%$ dans la région voisine de $120{ }^{\circ} \mathrm{C}$ puis croît fortement jusqu'à un maximum de $55 \%$. La fraction grasse évolue de $10 \%$ en entrée jusque $20 \%$ en sortie en passant par un maximum de $30 \%$ aux alentours de $120{ }^{\circ} \mathrm{C}$. La fraction carbohydratée évolue similairement à la fraction grasse avec des valeurs plus faibles : de $2 \%$ en entrée, elle évolue à $5 \%$ en sortie en passant par un maximum de $14 \%$. Les teneurs en matières sèches totales des dépôts frais recueillis dans les zones $\mathrm{E}$ et $\mathrm{F}$ sont voisines, environ $10 \%$ en zone $\mathrm{E}$ et $15 \%$ en zone $\mathrm{F}$.

TABleau 1

Composition moyenne des dépôts recueillis sur les surfaces d'échange thermique lors des différents essais

Mean composition of deposits collected on the heat exchange surfaces

\begin{tabular}{|c|c|c|c|c|c|c|c|}
\hline & \multirow{3}{*}{ COMPOSITION } & \multicolumn{6}{|c|}{ Zone de traitement thermique } \\
\hline & & \multicolumn{3}{|c|}{$\mathrm{E}$} & \multicolumn{3}{|c|}{$\mathrm{F}$} \\
\hline & & $70^{\circ} \mathrm{C}$ & $90^{\circ} \mathrm{C}$ & $110^{\circ} \mathrm{C}$ & $110^{\circ} \mathrm{C}$ & $120^{\circ} \mathrm{C}$ & $130^{\circ} \mathrm{C}$ \\
\hline \multicolumn{2}{|r|}{ EAU } & \multicolumn{3}{|c|}{$90 \%$} & \multicolumn{3}{|c|}{$85 \%$} \\
\hline \multirow{4}{*}{$\begin{array}{l}\text { Matières } \\
\text { sèches }\end{array}$} & Fraction protéique & 39 & 54 & 58 & 43 & 35 & 20 \\
\hline & Fraction grasse & 12 & 11 & 12 & 10 & 30 & 20 \\
\hline & Fraction minérale & 11 & 19 & 28 & 45 & 21 & 55 \\
\hline & Fraction carbohydrate & 38 & 16 & 2 & 2 & 14 & 5 \\
\hline
\end{tabular}

\section{B. Suivi de l'encrassement}

La figure 4 représente l'évolution des différents taux de colmatage des sections de l'appareil. En début d'essai, tant que de l'eau est encore en circulation, les valeurs de alpha sont toutes nulles. Le produit arrivant dans les différentes sections crée un décalage d'échelle des valeurs de alpha, dû aux 
propriétés physiques différentes du produit par rapport à l'eau (viscosité essentiellement). Une fois les valeurs restabilisées (échangeur totalement rempli et front eau-produit passé), on note une évolution régulière des valeurs d'encrassement en fonction du temps. Celle-ci correspond aux répartitions des dépôts observées dans les opérations de pesage des plaques. La zone F s'encrasse de façon la plus importante, la zone E faiblement et la zone $\mathrm{H}$ pas du tout. Le suivi d'encrassement étant possible par cette méthode pour les produits servant à la préparation de crèmes dessert chocolatées, l'installation d'un capteur industriel est en cours d'exécution sur une ligne de fabrication industrielle de ces produits.

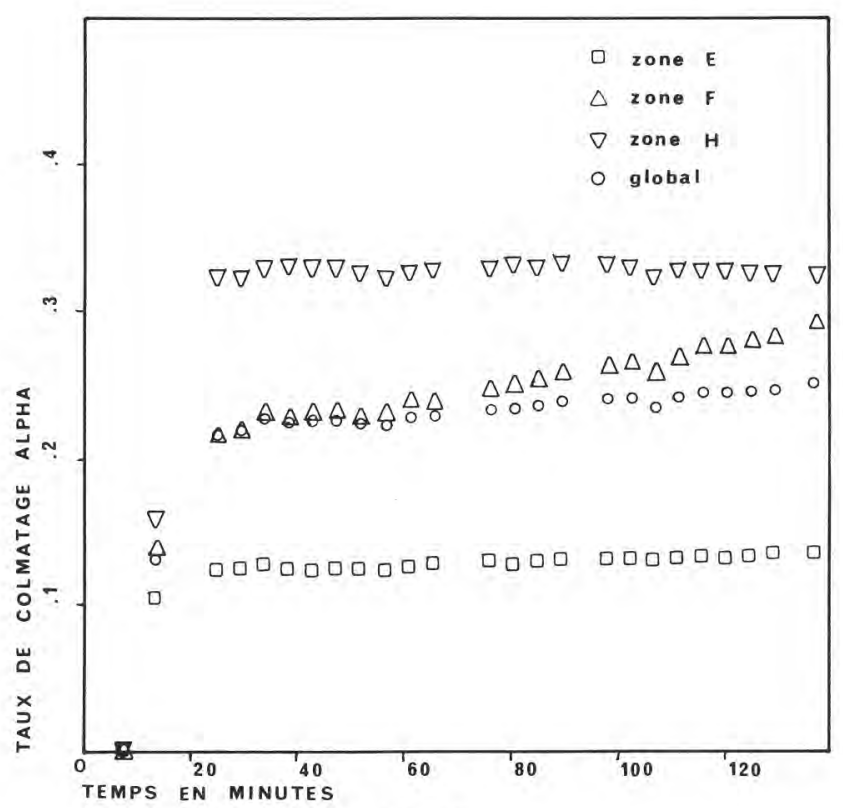

Fig. 4

Evolution du taux de colmatage $\alpha$ en fonction du temps pour les zones d'échanges thermiques $E, F$ et $H$ (essai $n^{o} 3$ ).

Fouling curves of the heat exchange sections $E, F$ and $H$ (trial $n^{o} 3$ ).

\section{Nettoyage}

Les résultats des séquences de nettoyage testées sont entièrement qualitatifs. Dans le cas de la stérilisation UHT de produits lactés chocolatés, le nettoyage de la section, notée $F$, n'est jamais total avec les agents classiques tels que la soude caustique et aboutit au démontage des installations pour une intervention manuelle. A la suite des trois essais d'encrassement réalisés, nous avons donc testé les séquences de nettoyage décrites au paragraphe I.C.5. Qualitativement, nous avons constaté après démontage que : 
- dans le cas de l'utilisation de soude caustique, l'échangeur présentait encore de grandes quantités de dépôts adhérant aux parois, surtout dans la zone $\mathrm{H}$ de stérilisation ;

- dans le cas du nettoyage effectué avec l'agent alcalin chloré, la quantité de dépôt restant dans l'échangeur était bien plus faible et plus clair que celui observé dans le cas précédent.

- dans le cas où la solution contenait l'agent oxydant, on note que la quantité de dépôt encore présente au démontage est faible et que les surfaces nettoyées présentent un aspect net et brillant.

Ces résultats qualitatifs nous ont conduit à réaliser des essais de nettoyage sur un appareil de type industriel instrumenté de façon à pouvoir suivre en continu l'évolution du taux de colmatage alpha. Cet appareil réalise les fonctions des zones $\mathrm{E}$ et $\mathrm{F}$ en une seule section sans récupération. Deux essais supplémentaires ont donc été mis en œuvre afin de tester l'efficacité réelle de ces produits dans le cas du nettoyage. Le nettoyage est effectué à l'aide d'une unité mobile programmable munie d'une pompe centrifuge dont le débit varie suivant l'état d'encrassement de l'appareil entre le débit de production de $12001 . h^{-1}$ et un maximum de $40001 . \mathrm{h}^{-1}$. Chaque essai est constitué de deux séquences. Le premier est effectué avec une solution à $1 \%(\mathrm{~kg} / \mathrm{kg})$ de l'agent alcalin chloré suivie d'une solution à $2,5 \%(\mathrm{~kg} / \mathrm{kg})$ de l'agent alcalin chloré additionnée à $0,5 \%(\mathrm{~kg} / \mathrm{kg})$ de l'agent oxydant (fig. 5). Le deuxième est effectué avec deux solutions à $2,5 \%(\mathrm{~kg} / \mathrm{kg})$ de l'agent alcalin chloré additionnée à $0,5 \%(\mathrm{~kg} / \mathrm{kg})$ de l'agent oxydant (fig. 5).

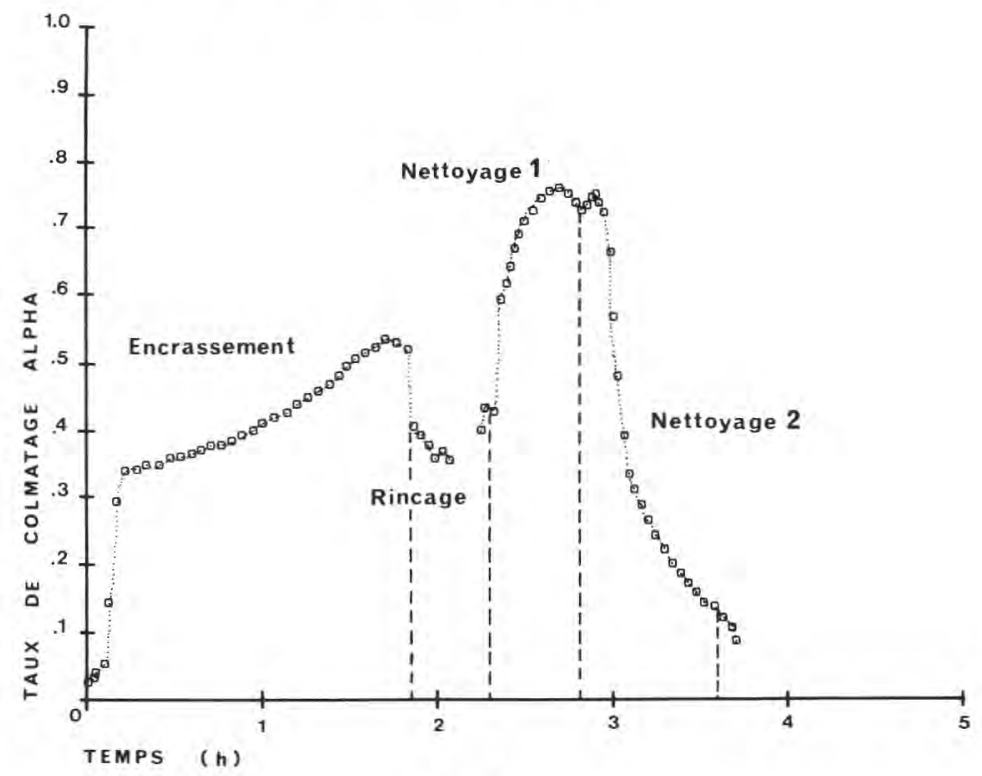

Fig. 5

Evolution du taux de colmatage $\alpha$ en fonction du temps lors de l'encrassement et du nettoyage d'un stérilisateur industriel. Mise en évidence du rôle nettoyant des produits testés.

Fouling and cleaning curve of an industrial sterilizer. Cleaning effect of the tested products. 


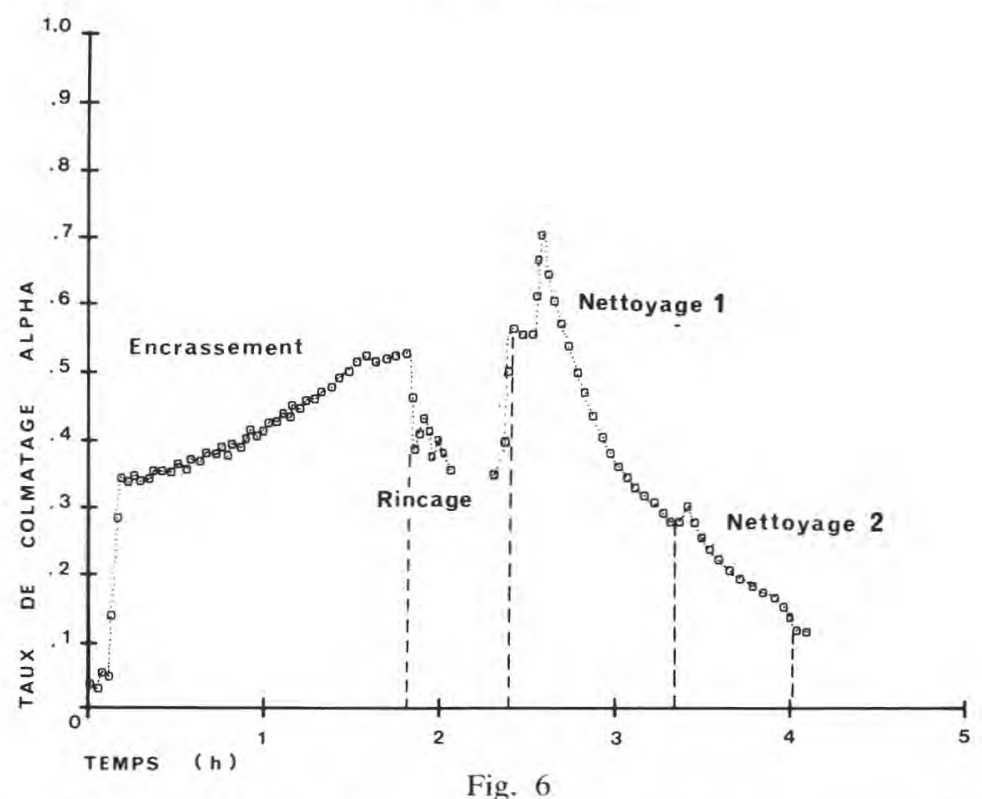

Evolution du taux de colmatage $\alpha$ en fonction du temps lors de l'encrassement et du nettoyage d'un stérilisateur industriel. Mise en évidence du rôle nettoyant des produits testés.

Fouling and cleaning curve of an industrial sterilizer. Cleaning effect of the tested products.

Plusieurs remarques se dégagent de ces essais. Tout d'abord, nous constatons que dans les deux cas, nous obtenons de réels profils de nettoyage, à savoir que l'on observe une diminution rapide de la valeur de alpha mesurée avec le temps de passage de la solution. Les valeurs finales obtenues sont très voisines de celles mesurées en début de manipulation sur eau. Après démontage de l'appareil, nous constatons que les plaques ont un aspect propre et brillant. Par endroit, nous notons encore la présence de souillures. Celles-ci se situent essentiellement au bord des joints de plaques, aux points de contact des plaques entre elles et dans les zones voisines des orifices d'admission.

Sur les figures 5 et 6 , il est clair que l'amélioration de l'efficacité du nettoyage est due à la combinaison des deux produits.

Sur la figure 5, la première séquence de nettoyage composée du produit alcalin seul dure 30 minutes. La courbe de alpha mesuré en fonction du temps passe par un maximum au bout de 20 minutes. On retrouve ici l'action classique des agents de nettoyage de type basique avec une première étape de gonflement du dépôt traduite par une croissance de la courbe $\alpha=f(t)$. Suit une période de 10 minutes pendant laquelle on note une décroissance de la courbe $\alpha=\mathrm{f}(\mathrm{t})$. Le temps programmé de 30 minutes étant atteint la solution est remplacée par la solution contenant le produit alcalin et l'agent oxydant. La courbe $\alpha=\mathrm{f}(\mathrm{t})$ devient de nouveau croissante pour passer rapidement par un maximum au bout de 4 minutes. On note alors une décroissance très rapide de cette courbe sur 20 minutes. Le rinçage sur eau final stabilise la valeur de alpha mesuré vers 0,091 pour une valeur de alpha de départ de 0,039. 
Sur la figure 6 , les deux séquences de nettoyage sont effectuées avec la combinaison des deux produits. La première séquence a été augmentée en temps et dure 40 minutes afin de mieux visualiser la courbe $\alpha=f(t)$ au cas où le même phénomène que celui de la figure 5 se présente. En fait, nous notons une croissance rapide de la courbe $\alpha=\mathrm{f}(\mathrm{t})$ pour atteindre un maximum au bout de 4 minutes. Ensuite, cette courbe décroît régulièrement de façon moins brutale que celle observée figure 5 dans la seconde séquence. Lorsque la seconde séquence se déclenche, on observe une nouvelle croissance de la courbe $\alpha=\mathrm{f}(\mathrm{t})$ qui passe par un maximum au bout de 4 minutes puis la courbe décroît régulièrement de façon identique à la $1^{\text {re }}$ séquence pendant encore 20 minutes. Dans ce cas-ci, le changement de séquence revient à utiliser dans les mêmes conditions une solution fraîche de produit. A la vue des courbes, on constate que cela n'a pas pour effet de donner un net accroissement d'efficacité. Le rinçage sur eau final stabilise la valeur de $\alpha$ mesuré vers 0,112 pour une valeur de $\alpha$ de départ de 0,040 .

Le rôle nettoyant des agents utilisés est bien mis en évidence par ces essais. Par comparaison des séquences employées, il est clair que la présence de l'agent oxydant joue un rôle efficace dans l'élimination des dépôts. Le temps nécessaire à l'obtention d'un maximum dans la courbe $\alpha=f(t)$ est considérablement réduit. Du point de vue du nettoyage global, on ne peut conclure sur une efficacité plus grande de l'une ou l'autre des méthodes employées, les valeurs finales de alpha obtenues sont voisines et atteintes dans le même temps de 80 minutes.

\section{Discussion}

A notre connaissance, l'étude de l'encrassement des plaques de stérilisateur UHT n'avait été réalisée que pour du lait (Burton, 1968; Tissier et al., 1984 ; Lalande et al., 1984) ou de la crème liquide (HidDinK et al., 1986 ; MaAs et al., 1985). Nous ne pouvons donc comparer nos résultats qu'à ceux de ces auteurs. Lors de l'encrassement d'une installation de stérilisation UHT par du lait, Tissier et al. (1984) notent que la zone comportant le volume le plus important de dépôt est la zone de préchauffage dont la température varie de $80^{\circ} \mathrm{C}$ à $120^{\circ} \mathrm{C}$, la zone de stérilisation contenant des volumes plus faibles de dépôt (température de $120^{\circ} \mathrm{C}$ à $138^{\circ} \mathrm{C}$ ). Dans notre cas, nous notons un phénomène inverse : le volume de dépôt présent dans la section $\mathrm{F}$ de stérilisation est beaucoup plus important que dans les autres zones. Indépendamment des proportions relatives de dépôts présents dans ces deux zones, nous pouvons observer que l'aspect de la courbe de répartition en masse (fig. 3) obtenue dans nos expérimentations est assez similaire à celle donnée par Tissier et al. (1984). De même, l'analyse des dépôts recueillis lors d'encrassement par du lait (Burton, 1968; Tissier et al., 1984) ou de la crème (Hiddink et al., 1986; MAAs et al., 1985) d'équipements de traitement thermique, indique que les compositions des dépôts sont voisines et évoluent de façon identique avec la température. Dans le cas de notre produit, la présence de carbohydrates modifie les valeurs des fractions protéiques, grasses et minérales mais les rapports de ces fractions entre elles restent voisins de celles données 
pour les dépôts du lait. On note que dans la zone de température variant de $70^{\circ} \mathrm{C}$ à $110^{\circ} \mathrm{C}$, le dépôt est à forte proportion protéique (aux environs de $50 \%$ ) et que dans la zone de température variant de $110^{\circ} \mathrm{C}$ à $130^{\circ} \mathrm{C}$, la fraction minérale du dépôt augmente fortement. D'autres remarques viennent compléter la similitude du phénomène d'encrassement avec celui observé dans le cas du lait : aucune des sections ayant une température inférieure à $70^{\circ} \mathrm{C}$ ne s'encrasse; chute très brutale de la quantité de dépôts formés entre les zones $\mathrm{F}$ et $\mathrm{H}$; encrassement des surfaces en zone $\mathrm{H}$ (récupération de température en phase descendante) très faible. Comme l'ont cité Tissier et al. (1984) et LALANDE et al. (1984), un prétraitement thermique du lait au voisinage de $80^{\circ} \mathrm{C}$ permet de réduire la quantité de dépôt formé dans la zone de température $80^{\circ} \mathrm{C}-120^{\circ} \mathrm{C}$. Dans notre procédé de fabrication, nous avons un chambrage de $5 \mathrm{mn}$ à $75^{\circ} \mathrm{C}$ avant l'entrée du produit en zone E. Si l'on considère que l'encrassement macroscopique, et en particulier sa croissance après que le phénomène ait été initié, est lié à la dénaturation de la $\beta$-lactoglobuline (LALANDE et al., 1985 ; RoIGNANT et al., 1986; DAUfin et al., 1987), on peut interpréter la répartition des dépôts observés dans cette zone comme le résultat d'une réaction chimique dans laquelle le produit réactif est fortement consommé durant le chambrage puis dont la concentration va en diminuant, l'effet d'élévation de température compensant légèrement la baisse de concentration du produit réactif. On observe dans ce cas, une répartition assez homogène de la quantité de dépôt avec un léger maximum en début de zone. Un ajustement numérique d'un tel modèle à nos résultats expérimentaux est actuellement en cours d'étude et nous permettra de conclure sur l'application, à notre produit, du phénomène décrit pour du lait (LALANDE et al., 1985).

L'interprétation de l'encrassement de la zone $\mathrm{F}$ de stérilisation paraît à l'heure actuelle assez délicate. La structure particulière du dépôt obtenu dans cette zone ne correspond pas à celle observée dans le cas du lait (BurTon, 1968 ; TiSSIER et al., 1984 ; LALANDE et al., 1984) ou de la crème (MAAS et al., 1985 ; Hiddink el al., 1986). Bien que les compositions déterminées pour les dépôts soient analogues, il semble que la présence de composés tels que l'amidon et le chocolat modifie les réactions et liaisons mises en jeu. On peut par exemple imaginer des phénomènes de cuisson donnant au dépôt la structure observée (réactions de Maillard, gélatinisation des amidons...).

Si l'on se réfère aux courbes d'encrassement obtenues (fig. 4), on note que l'encrassement des surfaces au cours du temps est un phénomène linéaire dans notre cas alors qu'il est présenté comme un phénomẽne proportionnel au carré du temps dans le cas du lait (LALANDE et al., 1984). Toutefois, les expérimentations faites avec du lait avaient été effectuées sur des durées de plusieurs heures (de 4 à 10 heures) qui permettent une appréciation plus sûre de l'évolution du phénomène. Dans notre cas, les durées de traitement thermique n'excèdent pas 2 h 30 . Si l'on compare les évolutions de l'encrassement sur la figure 4 , on note que l'encrassement prépondérant est celui de la zone F. Ceci est tout à fait en accord avec les répartitions en masse de dépôt observées. Le décalage des courbes par rapport au zéro contient un renseignement intéressant sur le produit. Ce décalage traduit la différence de propriétés physiques entre le produit et de l'eau en écoulement dans les mêmes conditions. Le produit subit au cours de son traitement des transformations qui font qu'entre les zones $\mathrm{E}, \mathrm{F}$ et $\mathrm{H}$, le décalage s'accroît. La mesure du taux de colmatage global établit une moyenne à la fois de ce décalage et des évolu- 
tions du taux de colmatage de chaque zone. Les durées de ces essais ne permettent pas d'obtenir des évolutions du taux de colmatage significatives en zone $\mathrm{E}$ pour établir si le phénomène est linéaire avec le temps ou proportionnel à son carré comme dans le cas du lait.

Dans le cas de la formation du dépôt en zone $\mathrm{F}$, la linéarité du phénomène avec le temps implique que l'encrassement ne dépend que des quantités de produit présentes dans la solution. Actuellement, nous ne sommes pas en état d'indiquer quel produit incriminer ni dans quel type de réaction il intervient.

\section{Conclusion}

Le problème technique majeur auquel sont soumis les échangeurs de chaleur à plaques servant à la fabrication et à la stérilisation des mélanges pour crèmes dessert est l'encrassement des surfaces et leur nettoyage. L'analyse qui a été faite pendant ces opérations nous apporte plusieurs éléments utiles pour améliorer le fonctionnement de ces stérilisateurs. Les composants principaux retrouvés dans les dépôts formés sont les protéines et les minéraux présents dans la préparation. La température joue un rôle prépondérant sur la quantité et la composition des dépôts recueillis. Les dépôts formés à des températures inférieures à $105-110^{\circ} \mathrm{C}$ sont à forte proportion protéique et s'apparentent à ceux que l'on peut observer lors du traitement thermique de lait ou de crème dans les mêmes conditions. Le chambrage effectué à $75^{\circ} \mathrm{C}$ explique suivant le même raisonnement l'allure uniforme de la répartition des dépôts en zone E. La dénaturation des protéines au voisinage de la paroi peut être envisagée comme facteur prépondérant de la cinétique d'encrassement. Le nettoyage de cette zone ne posait pas dans nos expérimentations de problème majeur. L'analyse du phénomène est plus délicate dans la zone de température de stérilisation entre 110 et $130^{\circ} \mathrm{C}$. On note que la fraction minérale du dépôt augmente dans cette zone de façon importante. Des phénomènes complexes d'interaction entre les différents composés présents confèrent au dépôt la structure particulière que l'on observe. En conséquence, nous ne pouvons proposer, pour l'instant et sans études ultérieures, d'interprétation de la cinétique d'encrassement observée. Pour ce qui concerne le nettoyage de ces dépôts, le problème de leur élimination reste ouvert. Pour une finalité industrielle, l'expérimentation sera poursuivie en vraie grandeur dans une usine de production. En effet, l'utilisation de produits élaborés pour le nettoyage augmente le coût de fonctionnement d'une ligne de fabrication. Le critère économique devant être introduit, il est indispensable de réaliser sur site une analyse économique permettant de mettre en œuvre la solution technique appropriée.

Reçu le 11 décembre 1986. Accepté pour publication le 26 mars 1987.

\section{Remerciements}

Nous remercions J.P. TISSIER, P. DEBREYNE, D. LOONIS et G. RONSE du Laboratoire de Génie Industriel Alimentaire pour leur collaboration technique lors du déroulement de ces essais. 


\section{Références bibliographiques}

Burton H., 1968. Deposits from whole milk in heat treatment plant : a review and discussion. $J$. Dairy Res., 35, 317-330.

COOPER A., 1974. Recover more heat with plate heat exchangers. Chem. Eng., May, 280-285.

Corrieu G., Lalande M., Ferret R., 1985. Mesure en ligne de l'encrassement et du nettoyage d'un stérilisateur UHT industriel. J. Food Eng., 5, 231-348.

Daufin G., labbé J.P., Quemerais A., Brulé G., Michel. F., Roignant M., Priol M., 1987. Fouling of a heat exchange surface by whey, milk and model fluids. An analytical study. Lait, $67,339-364$.

Delsal J.L., 1944. Nouvelle procédure d'extraction des lipides du sérum par le méthytal. Bull. Soc. Chim. Biol., 26, 99-105.

Dubois M., Gulles K.A., Hamilton J.K., Rerers P.A., Sмith, 1956. Colorimetric method for determination of sugars and related substances. Anal. Chem., 28, 350-356.

Helaine E., 1977. Dosage de l'ammoniac dans le lait par électrode spécifique. Ind. Alim. Agric., (6), $581-588$

Hiddink J., Lalande M., Maas A.J.R., Streuper A., 1986. Heat treatment of whipping cream. 1. Fouling of the pasteurization equipment. Milchwissenschaft, 41 (9), 542-546.

Lalande M., Corrieu G., 1979. Procédé de contrôle de l'encrassement et de décrassage d'un matériel. Appareil de mesure du degré d'encrassement d'un matériel. Brevet français n" 1979/ 09.969.

Lalande M., Corrieu G., Tissier J.P., Ferret R., 1979. Etude du comportement d'un échangeur à plaques VICARB utilisé pour la pasteurisation du lait. Lait, 59, 13-33.

Lalande M., Tissier J.P., Corrieu G., 1984. Fouling of a plate heat exchanger used in ultra temperature sterilization of milk. J. Dairy Res., 51, 123-142.

Lalande M., Tissier J.P., Corrieu G., 1985. Fouling of heat transfer surfaces related to $\beta$ lactoglobulin denaturation during heat processing of milk. Biotechnol. Prog., I (2), 131-139.

Lund D.B., Bıxвy D., 1975. Fouling of heat exchange surfaces by milk. Process Biochem., 10 (9), 52-55.

LySTER R.L.J., 1965. The composition of milk deposits in a UHT plant. J. Dairy Res., 32, 203208.

MaAs R., Lalande M., Hiddink J., 1985. Fouling of a plate heat exchanger by whipping cream. In : Fouling and cleaning in Food Processing. Ed. Lund D., Plett E. and Sandu C., University of Madison, Wisconsin, USA, 217-225.

Marriot J., 1971. Where and how to use plate heat exchangers. Chem. Eng., 5, 127-134.

Palller F.M., 1982. Dosage après minéralisation des protéines par électrode spécifique de l'ion ammonium. Comparaison avec la méthode de KJELDAHL, application aux denrées alimentaires. Ann. Falsif. Expert. Chim., 75 (811), 431-439.

Perlat M.N., Lalande M., Corrieu G., 1986. Etude de nettoyage d'un stérilisateur de lait UHT. Ordre d'utilisation des détergents alcalin et acide et aspects cinétiques, Lail, 66, 31-63.

RENÉ F., 1985. Contribution à l'étude de l'encrassement et du nettoyage d'un échangeur de chaleur à plaques. DEA, ENSIA, Massy.

Roignant M., Daufin G., Michel. F., Kerherve F.L., Brulé G., 1986. Traitement thermique du rétentat $(\times 2)$ d'ultrafiltration de lait dans un échangeur à plaques: aspects thermiques, hydrodynamiques et encrassement par comparaison au lait et au lactosérum. Lait, 66 (3), 207 232.

Tissier J.P., Lalande M., Corrieu G, 1984. A study of milk deposit on heat exchange surface during UHT treatment. Eng. Food, 1 (6), 49-58.

VinconNeAu M., CREvel M., 1976. Les échangeurs à plaques et à spirales dans l'industrie sucrière. Ind. Alim. Agric., 825-833. 